\title{
Parasites gastro-intestinaux chez les chiens à Lubumbashi
}

\author{
Daudet Byakya ${ }^{1 *}$ Boniface Lombe ${ }^{1}$ \\ Yan Madimba ${ }^{2}$ Emmanuel Kaluendi ${ }^{3}$
}

\section{Mots-clés}

Chien, helminthe, protozoa, parasitisme, République démocratique du Congo

Submitted: 14 April 2018

Accepted: 13 November 2018

Published: 14 January 2019

DOI: $10.19182 /$ remvt.31668

\section{Résumé}

Une étude parasitologique a été menée en 2015 sur des chiens de sexe, âge et race différents, vivant en liberté dans la ville de Lubumbashi, République démocratique du Congo. Des échantillons de fèces ont été prélevés et analysés par coproscopie selon une méthode de flottation. Sur les 120 chiens examinés, 78 (65\%) étaient infestés, parmi lesquels $74(61,7 \%)$ avaient des helminthes gastro-intestinaux et $4(3,3 \%)$ avaient des coccidies. Les coproscopies ont révélé la présence de cinq espèces de parasites : Ankylostoma spp. (41 chiens), Toxocara canis (22), Toxascaris leonina (8), Isospora canis (4) et Dipylidium caninum (3). I. canis n'a été retrouvé que chez les chiots. Le dépistage fréquent d'ankylostomes et de $T$. canis suggère qu'il existe à Lubumbashi un risque de contamination de I'homme par ces parasites zoonotiques.

- Pour citer cet article : Byakya D., Lombe B., Madimba Y., Kaluendi E., 2018. Gastrointestinal parasites in dogs in Lubumbashi. Rev. Elev. Med. Vet. Pays Trop., 71 (4): 173-176, doi: 10.19182/remvt.31668

\section{INTRODUCTION}

En République démocratique du Congo (RDC), comme partout en Afrique, le chien est principalement élevé pour assurer la protection des personnes et de leurs biens. Ce service offre au chien le privilège de vivre dans des rapports étroits avec les humains. Cependant, la coexistence n'est pas sans risque pour ces derniers. Outre les cas de morsures, le chien peut présenter un danger pour la santé humaine (Euzéby, 1966) car il constitue une source potentielle de zoonoses. Il peut être parasité par de nombreuses espèces d'helminthes et de protozoaires (Lamy, 1980). En l'absence de traitements antiparasitaires, les chiens constituent des réservoirs de parasites zoonotiques. La prévalence chez le chien est favorisée surtout par le manque d'hygiène,

1. Laboratoire vétérinaire, Kinshasa, République démocratique du Congo.

2. Université de Kinshasa, Faculté de médecine vétérinaire, Kinshasa, République démocratique du Congo.

3. Université de Lubumbashi, Faculté de médecine vétérinaire, Lubumbashi, République démocratique du Congo.

* Auteur pour la correspondance

Tél. : +243823343518/842162289

E-mail :dbyakya@gmail.com et la prévalence de zoonoses est alors favorisée par les contacts entre l'homme et l'animal (Coggins, 1998). Parmi les parasites zoonotiques présents chez le chien, les plus importants sont Ankylostoma spp. et Toxocara canis (Kahn et Line, 2004), qui affectent principalement les enfants âgés d'un à trois ans (Overgaauw et van Knapen, 2008). Le climat chaud et humide en RDC est particulièrement propice au développement des cycles parasitaires. L'objectif de la présente étude a été de déterminer la prévalence du parasitisme digestif dans une population de chiens domestiques de Lubumbashi.

\section{MATERIEL ET METHODES}

\section{Zone d'étude}

L'enquête a été réalisée dans les sept communes de la ville de Lubumbashi (Lubumbashi, Kamalondo, Kenya, Katuba, Kampemba, Ruashi et Annexes). Lubumbashi est située au sud-est de la RDC dans la province de Haut-Katanga, à une altitude de 1250 mètres, à $27^{\circ} 28^{\prime} \mathrm{E}$ et $11^{\circ} 40^{\prime} \mathrm{S}$. Le climat est tropical humide avec une saison des pluies de six mois (novembre à mars) et une saison sèche de six mois (avril à octobre). La température annuelle moyenne est de $20^{\circ} \mathrm{C}$, la pluviométrie totale annuelle moyenne est de $1230 \mathrm{~mm}$, janvier et février étant les mois les plus pluvieux. L'humidité relative journalière moyenne est de $60 \%$ (Assani, 1999). 
A Lubumbashi, le mode de vie des chiens est de type traditionnel : ils sont en liberté, laissés en divagation toute la journée, nourris avec les restes de repas, et ne bénéficient d'aucun soin. Cependant, quelques familles élèvent des chiens, en majorité de race exotique, dans de bonnes conditions sanitaires, leur réservant des niches, de la nourriture appropriée, et leur prodiguant des soins réguliers. Chez ces familles, les chiens restent dans leur niche pendant la journée et sont libérés la nuit dans les parcelles à des fins de gardiennage.

\section{Animaux}

L'effectif a porté sur 120 chiens de sexe, âge et race différents, sélectionnés au hasard, dont la répartition est décrite dans le tableau I.

\section{Méthodes}

Des échantillons de fèces ont été récoltés entre $6 \mathrm{~h}$ et $10 \mathrm{~h}$ pendant trois semaines, du 25 avril au 16 mai 2015. Les matières fécales ont été collectées par voie rectale chez les chiens adultes, à l'aide d'un ou deux doigts gantés selon la taille de l'animal ou chez les chiots âgés de moins de 18 mois à l'aide d'un coton-tige. Les échantillons ont été transportés dans une glacière pour être analysés immédiatement - ou conservés moins de 24 heures au réfrigérateur à $4{ }^{\circ} \mathrm{C}$ - au laboratoire de parasitologie de la Faculté de médecine vétérinaire de l'Université de Lubumbashi.

Les coproscopies qualitatives ont été réalisées par flottation en saumure préparée en dissolvant $400 \mathrm{~g}$ de sel de cuisine dans un litre d'eau. Cette saumure (densité de 1,19 à $20^{\circ} \mathrm{C}$ ) convient pour dépister rapidement les nématodes et les cestodes. Le mélange analysé était constitué de $2 \mathrm{~g}$ de fèces pour $100 \mathrm{ml}$ de solution (Talvik et al., 2006).

\section{Tableau I}

Répartition des chiens examinés dans I'agglomération de Lubumbashi, République démocratique du Congo

$\begin{array}{lcccc}\text { Commune } & \text { Chiots } & \text { Chiennes } & \text { Chiens } & \text { Total } \\ \text { Annexes } & 3 & 5 & 8 & 16 \\ \text { Kamalondo } & 2 & 10 & 5 & 17 \\ \text { Kampemba } & 4 & 6 & 9 & 19 \\ \text { Katuba } & 5 & 7 & 2 & 14 \\ \text { Kenya } & 6 & 1 & 9 & 16 \\ \text { Lubumbashi } & 5 & 8 & 7 & 20 \\ \text { Ruashi } & 6 & 8 & 4 & 18 \\ \text { Total } & 31 & 45 & 44 & 120\end{array}$

La suspension a été filtrée sur un tamis nettoyé avant toute nouvelle manipulation. Le filtrat a été versé dans un tube à essai de $5 \mathrm{ml}$ rempli au maximum et recouvert d'une lamelle, puis centrifugé à 2500 tours/ min pendant $5 \mathrm{~min}$. La lamelle a été déposée sur une lame identifiée au nom du prélèvement, avant d'être observée au microscope. Les prélèvements ont été observés à un faible grossissement $(\times 40)$, afin de rechercher les œufs et les larves présents dans les échantillons, puis à un fort grossissement $(\times 100$ et $\times 400)$, pour préciser leur identification.

\section{Analyse statistique}

Les résultats des examens coprologiques ont été analysés par le test de comparaison de prévalence ou Chi carré.

\section{RESULTATS}

Sur les 120 chiens examinés, 78 (65\%) étaient infestés dont 74 $(61,7 \%)$ par des helminthes gastro-intestinaux de quatre espèces différentes (tableau II). Quatre chiens étaient par ailleurs infestés par la coccidie Isospora canis.

Les trois groupes d'animaux étaient infestés par les helminthes mais les taux d'infestation n'étaient pas significativement différents $\left(\mathrm{Chi}^{2}\right.$ $=3,17 ; p=0,21$ ). Il n'y a pas eu non plus de différence entre les sexes. Tous les sexes et âges ont été trouvés infestés par Ankylostoma spp., Toxocara canis et Toxascaris leonina, tandis que I. canis n'a été rencontré que chez les chiots.

En revanche, des différences significatives d'infestation $\left(\mathrm{Chi}^{2}=19,3\right.$; $p=0,004)$ ont été observées entre les communes, les infestations ayant été plus fréquemment diagnostiquées à Katuba, Kamalondo, Annexes et Ruashi (tableau III). La commune la plus infestée a été Katuba et la moins Kampemba.

\section{DISCUSSION}

Le chien est souvent infesté, parfois de façon massive, par de nombreuses espèces d'helminthes. Plusieurs de ces espèces sont aussi observées chez les carnivores sauvages (Bwalya et al., 2011). La viande, les poissons crus et surtout les déchets d'organes constituent la source d'infestation la plus importante pour les cestodes (Taenia, Echinococcus) et les trématodes (Thienpont et al., 1979) alors que l'infestation par les nématodes provient principalement de l'ingestion d'œufs ou de larves infestantes présents dans l'environnement.

Cette étude a montré que l'infestation la plus fréquente des chiens de Lubumbashi était celle par Ankylostoma spp. suivie de celle par Toxocara canis. Beaucoup d'études ont aussi révélé la forte

\section{Tableau II}

Infestation des chiens par des parasites gastro-intestinaux selon I'âge et le sexe dans I'agglomération de Lubumbashi, République démocratique du Congo

\begin{tabular}{lccccccc} 
Catégorie & Examinés & $\begin{array}{c}\text { Infestés } \\
(\%)\end{array}$ & $\begin{array}{c}\text { Ankylostoma } \\
\mathbf{s p p} \\
(\%)\end{array}$ & $\begin{array}{c}\text { Dipylidium } \\
\text { caninum } \\
(\%)\end{array}$ & $\begin{array}{c}\text { Isospora } \\
\text { canis } \\
(\%)\end{array}$ & $\begin{array}{c}\text { Toxascaris } \\
\text { leonina } \\
(\%)\end{array}$ & $\begin{array}{c}\text { Toxocara } \\
\text { canis } \\
(\%)\end{array}$ \\
\hline Chiots & 31 & $24(77,4)$ & $2(6,4)$ & $1(3,2)$ & $4(12,9)$ & $5(16,1)$ & $12(38,7)$ \\
Chiennes & 45 & $26(57,8)$ & $20(44,4)$ & - & - & $2(4,4)$ & $4(8,9)$ \\
Chiens & 44 & $28(63,6)$ & $19(43,2)$ & $2(4,5)$ & - & $1(2,3)$ & $6(13,6)$ \\
\hline Total & 120 & $78(65)$ & $41(34,2)$ & $3(2,5)$ & $4(3,3)$ & $8(6,7)$ & $22(18,3)$
\end{tabular}




\section{Tableau III}

Infestation de chiens par des parasites gastro-intestinaux selon les communes dans l'agglomération de Lubumbashi, République démocratique du Congo

\begin{tabular}{|c|c|c|c|c|c|c|c|}
\hline $\begin{array}{l}\text { Site de } \\
\text { prélèvement }\end{array}$ & Examinés & $\begin{array}{c}\text { Infestés } \\
(\%)\end{array}$ & $\begin{array}{c}\text { Ankylostoma } \\
\text { spp. } \\
(\%)\end{array}$ & $\begin{array}{c}\text { Dipylidium } \\
\text { caninum } \\
(\%)\end{array}$ & $\begin{array}{c}\text { Isospora } \\
\text { canis } \\
(\%)\end{array}$ & $\begin{array}{c}\text { Toxascaris } \\
\text { Ieonina } \\
(\%)\end{array}$ & $\begin{array}{c}\text { Toxocara } \\
\text { canis } \\
(\%)\end{array}$ \\
\hline Katuba & 14 & $13(92,9)$ & $7(50,0)$ & $2(14,3)$ & - & $1(7,1)$ & $3(21,4)$ \\
\hline Kamalondo & 17 & $15(88,2)$ & $10(58,8)$ & - & - & $3(17,7)$ & $2(11,8)$ \\
\hline Annexes & 16 & $13(81,3)$ & $7(43,8)$ & $1(6,2)$ & - & - & $5(31,2)$ \\
\hline Ruashi & 18 & $12(66,7)$ & $4(22,2)$ & - & $2(11,1)$ & - & $6(33,3)$ \\
\hline Kenya & 16 & $8(50,0)$ & $3(18,8)$ & - & - & $2(12,5)$ & $3(18,8)$ \\
\hline Lubumbashi & 20 & $9(45,0)$ & $6(30,0)$ & - & $1(5,0)$ & - & $2(10,0)$ \\
\hline Kampemba & 19 & $8(42,1)$ & $4(21,0)$ & - & $1(5,2)$ & $2(10,5)$ & $1(5,2)$ \\
\hline Total & 120 & $78(65)$ & $41(34,1)$ & $3(2,5)$ & $4(3,3)$ & $8(6,7)$ & $22(18,3)$ \\
\hline
\end{tabular}

prévalence d'Ankylostoma spp. lors d'examen coprologique chez les chiens à Lubumbashi (Makumyaviri et Suila, 1998), au Nigeria (Ajayi et al., 2000), au Zimbabwe (Mukaratirwa et Busayi, 1995) et en Inde (Traub et al., 2005). Le mode de vie des chiens de Lubumbashi contribue à la diffusion permanente d'œufs d'ankylostomes et d'ascarides et les prédispose ainsi aux réinfestations par ces nématodes. Ankylostoma caninum est la principale cause de l'ankylostomose canine dans la plupart des régions tropicales et subtropicales du monde (Barutzki et Schaper, 2003), mais la coproscopie ne permet pas de faire une identification précise d'espèce entre $A$. caninum, $A$. braziliense et Uncinaria stenocephala, les œufs de ces trois nématodes étant très similaires. Leurs larves infestantes peuvent entrer et errer sous la peau ou dans le corps humain, et provoquer soit une larva migrans cutanée, soit une entérite éosinophilique (Overgaauw et Van Knapen, 2008).

Le taux d'infestation par Toxocara canis était de 18,3\%, comparable à celui déjà observé dans cette même ville en 1998 (Makumyaviri et Suila, 1998). L'infestation par T. canis est grave chez le chien et cette espèce peut provoquer un syndrome de larva migrans viscérale lorsque les œufs embryonnés sont ingérés par des humains. Des larves peuvent se loger dans la rétine, provoquant une larva migrans oculaire (Normand et al., 2006) qui constitue un danger potentiel, spécialement chez les enfants âgés d'un à quatre ans (Overgaauw et Van Knapen, 2008). Dipylidium caninum a été retrouvé chez 2,5 \% des chiens mais il faut noter que la coproscopie par flottation a une sensibilité très faible pour le diagnostic de cestodoses. En effet les œufs sont rarement présents dans les fèces, et les segments ovigères sont souvent éliminés en dehors des défécations. Ceci peut expliquer la faible prévalence de taeniidés dans notre étude. Isospora canis est un agent de coccidiose canine, une pathologie dont les signes majeurs sont l'entérite diarrhéique et la dysenterie (selles hémorragiques) chez les jeunes chiens (Huart et Tshibangu, 1994 ; Overgaauw et Van Knapen, 2013).

A Lubumbashi, les chiens divagants et en quête d'immondices pour se nourrir représentent des sources de parasites. La faible médicalisation des chiens de propriétaires est également un facteur favorisant les parasitoses. Dans la présente étude, les variations significatives des taux d'infestation entre les différentes communes pouvaient être liées à l'état de salubrité publique dont l'entretien variait d'un quartier à l'autre (Sager et al., 2006). Ainsi, les taux d'infestation ont été plus élevés à Katuba, Kamalondo, Annexes et Ruashi, où les immondices et les mares sont plus présents.

L'importance des infestations parasitaires dans la population canine, associée au risque zoonotique, fait des helminthoses intestinales animales un problème sanitaire majeur. L'amélioration des conditions de vie des chiens et de leur taux de médicalisation, incluant des vermifugations régulières, réduirait les taux d'infestation dans les villages d'Afrique (ESCCAP, 2010 ; Strube et al., 2013). Toutefois, compte tenu des conditions socioéconomiques, il paraît difficile d'utiliser régulièrement des vermifuges pour les chiens alors même que les villageois n'en disposent pas pour eux-mêmes.

\section{CONCLUSION}

La détection d'Ankylostoma spp., de Toxocara canis, Toxascaris leonina et Dipylidium caninum chez les chiens à Lubumbashi indique un risque de contamination des humains par les ankylostomes et les ascarides. Pour un meilleur contrôle du parasitisme gastro-intestinal des chiens, il faudrait : a) éviter leur divagation ; b) contrôler les infestations parasitaires par la vermifugation tout au long de leur vie ; et c) appliquer un contrôle strict de l'alimentation des animaux de compagnie. Les propriétaires de carnivores domestiques doivent être informés sur les risques potentiels d'une infestation parasitaire, non seulement pour la santé de leurs animaux, mais également pour celle de leur famille et de toute personne en contact direct ou indirect avec leurs animaux.

\section{REFERENCES}

Ajayi O.O., Duhlinska D.D., Agwale SM., Njoku M., 2000. Frequency of human toxocariasis in Jos, Plateau State, Nigeria. Mem. Inst. Oswaldo Cruz Rio de Janeiro, 95 (2): 147-149, doi: 10.1590/S0074-02762000000200002

Assani A.A., 1999. Variabilité temporelle et persistance des épisodes secs en saison de pluies à Lubumbashi (Congo-Kinshasa). Sécheresse, 10 (1) : 45-53

Barutzki D., Schaper R., 2003. Endoparasites in dogs and cats in Germany 19992002. Parasitol. Res., 90 (S3): S148-S150, doi: 10.1007/s00436-003-0922-6.

Bwalya E.C., Nalubamba K.S., Hankanga C., Namangala B., 2011. Prevalence of canine gastrointestinal helminths in urban Lusaka and rural Katete Districts of Zambia. Prev. Vet. Med., 100 (3-4): 252-255, doi: 10.1016/j. prevetmed.2011.04.015

Coggins J.R., 1998. Effect of season, sex, and age on prevalence of parasitism in dogs from Southeastern Wisconsin. J. Helminthol. Soc. Wash., 65 (2): 219-224

ESCCAP (European Scientific Counsel for Companion Animal Parasites), 2010. Worm control in dogs and cats, Guideline 01, 2nd Edn., www.esccap.org

Euzéby J., 1966. Les maladies vermineuses des animaux domestiques et leurs incidences sur la pathologie humaine ; tome II, Maladies dues aux némathelminthes ; tome III, Maladies dues aux plathelminthes. Vigot Frères Paris, France, $798 \mathrm{p}$ 
Huart A., Tshibangu M., 1994. Pathologie des petits animaux. Rec. Méd. Vét., 8 (4) $17-21$

Kahn C.M., Line S., 2004. The Merck veterinary manual, $9^{\text {th }}$ Edn. Merck, New York, USA, 2712 p.

Lamy L.H., 1980. Protozoaires et helminthes parasites : recherche et identification au laboratoire. Maloine, Paris, France, 622 p.

Makumyaviri A.M., Suila R., 1998. Dominante étiologique des helminthoses gastro-intestinales chez les chiens élevés dans la ville de Lubumbashi. Ann. Vét., 14 (9) : 34-41

Mukaratirwa S., Busayi R.M., 1995. A survey of patent gastrointestinal parasites of stray dogs in Bulawayo urban area. Zimb. Vet. J., 26 (1): 19-27

Normand T., Bourry O., Dang H., Leroy E., Bourdoiseau G., Davoust B., 2006. Enquête sur le parasitisme digestif des chiens dans une zone rurale du Gabon. Bull. Acad. Vét. France, 159 (1) : 59-67, doi: 10.4267/2042/47813

Overgaauw P.A.M., van Knapen F., 2008. Toxocarosis, an important zoonosis. Eur. J. Companion Anim. Pract., 18 (3): 259-266

\section{Summary}

Byakya D., Lombe B., Madimba Y., Kaluendi E. Gastrointestinal parasites in dogs in Lubumbashi

A parasitological study was conducted in 2015 in dogs of different sexes, ages and breeds, living freely in the city of Lubumbashi, Democratic Republic of the Congo. A coprological examination of the fecal samples was carried out with the flotation technique. Of the 120 dogs examined, 78 (65\%) were infested, of which $74(61.7 \%)$ had gastrointestinal helminths and $4(3.3 \%)$ had coccidia. Results revealed the presence of five parasite species: Ankylostoma spp. (41 dogs), Toxocara canis (22), Toxascaris leonina (8), Isospora canis (4) and Dipylidium caninum (3). I. canis was only found in puppies. The frequent detection of hookworms and T. canis suggests that there is a risk of human contamination by these zoonotic parasites in Lubumbashi.

Keywords: dogs, helminths, protozoa, parasitism, Democratic Republic of the Congo
Overgaauw P.A.M., van Knapen F., 2013. Veterinary and public health aspects of Toxocara spp. Vet. Parasitol, 193 (4): 398-403, doi: 10.1016/j.vetpar.2012. 12.035

Sager H., Steiner Moret C., Grimm F., Deplazes P., Doherr M.G., Gottstein B., 2006. Coprological study on intestinal helminths in Swiss dogs: temporal aspects of anthelminthic treatment. Parasitol. Res., 98 (4): 333-338, doi: 10.1007/s00436-005-0093-8

Strube C., Heuer L., Janecek E., 2013. Toxocara spp. infections in paratenic hosts. Vet. Parasitol. 193 (4): 375-389, doi: 10.1016/j.vetpar.2012.12.033

Talvik H., Moks E., Mägi E., Järvis T., Miller I., 2006. Distribution of Toxocara infection in the environment and in definitive and paratenic hosts in Estonia. Acta Vet. Hung., 54 (3): 399-406, doi: 10.1556/AVet.54.2006.3.10

Thienpont D., Rochette F., Vanparijs O., 1979. Diagnostic de verminose par examen coprologique. Janssen Research Foundation, Brussels, Belgium, 187 p.

Traub R.J., Robertson I.D., Irwin P.J., Mencke N., Thompson R.C., 2005. Canine gastrointestinal parasitic zoonoses in India. Trends Parasitol., 21 (1): 42-48, doi: 10.1016/j.pt.2004.10.011

\section{Resumen}

Byakya D., Lombe B., Madimba Y., Kaluendi E. Parásitos gastrointestinales en perros en Lubumbashi

Se llevó a cabo un estudio parasitológico, en 2015, en perros de diferentes sexos, edades y razas, viviendo libremente en la ciudad de Lubumbashi, República Democrática del Congo. Un examen coprológico de las muestras fecales se llevó a cabo mediante el método de flotación. De los 120 perros examinados, $78(65 \%)$ estaban infestados, de los cuáles 74 $(61,7 \%)$ tenían helmintos gastrointestinales y 4 (3,3\%) tenían coccidia. Los resultados revelan la presencia de cinco especies de parásitos: Ankylostoma spp. (41 perros), Toxocara canis (22), Toxascaris leonina (8), Isospora canis (4) y Dipylidium caninum (3). I. canis se encontró solamente en cachorros. La detección frecuente de anquilostomas y $T$ canis sugiere que existe un riesgo de contaminación a humanos de estos parásitos zoonóticos en Lumbashi.

Palabras clave: perro, helminto, protozoo, parasitismo, República democrática del Congo 